\title{
Expression of VEGF, CD73 and their relationship with clinical pathology, microvessel density, and prognosis in renal cell carcinoma
}

\author{
Xuefeng Mei ${ }^{1 \#}$, Jia Shu ${ }^{2 \#}$, Ruizhen Huang ${ }^{3 \#}, \mathrm{Xin} \mathrm{Chu}^{4}$, Ying Tian ${ }^{1}$ \\ ${ }^{1}$ Department of Urology, ${ }^{2}$ Department of Functional Inspection, ${ }^{3}$ Department of Cardiology, ${ }^{4}$ Nursing Department, Affiliated Hospital of Chengdu \\ University of Traditional Chinese Medicine, Chengdu, China \\ Contributions: (I) Conception and design: X Mei, J Shu, R Huang, Y Tian; (II) Administrative support: X Chu; (III) Provision of study materials or \\ patients: X Mei, J Shu, X Chu; (IV) Collection and assembly of data: All authors; (V) Data analysis and interpretation: X Mei, J Shu, R Huang, Y \\ Tian; (VI) Manuscript writing: All authors; (VII) Final approval of manuscript: All authors. \\ \#These authors contributed equally to this work. \\ Correspondence to: Ying Tian. Department of Urology, Hospital of Chengdu University of Traditional Chinese Medicine, No. 39 Shi-er-qiao Road, \\ Chengdu, China. Email: tianying810516@126.com.
}

Background: Renal cell carcinoma (RCC), a tumor originating from renal tubular epithelial cells, has the second highest incidence of all adult urogenital tumors. However, in most cases, patients show no obvious symptoms in the early or even the late stages of RCC, which seriously impacts the prognosis. This study aimed to analyze the expression of vascular endothelial growth factor (VEGF) and ecto-5'-nucleotidase (CD73) and their relationship with clinical pathology, microvessel density (MVD), and prognosis in RCC.

Methods: The clinical data of 76 patients with RCC who underwent radical nephrectomy in our hospital between October, 2011 and October, 2013 were retrospectively analyzed. The postoperative paraffin specimens were collected; the expression levels of VEGF and CD73 in the tumor tissues were detected, and the MVD was measured. T the expression of VEGF and CD73 and their relationship with clinical pathology, MVD, and prognosis in RCC.

Results: The positive expression rates of VEGF and CD73 in RCC patients with grades G3-G4 were higher than those in patients with grades $\mathrm{G} 1-\mathrm{G} 2(\mathrm{P}<0.05)$. The rates in RCC patients with stages III IV were higher than those in patients with stages $\mathrm{I}-\mathrm{II}(\mathrm{P}<0.05)$. The rates in RCC patients with lymph node metastasis were higher than those in patients without lymph node metastasis $(\mathrm{P}<0.05)$. The MVD count of patients with positive expressions of VEGF and CD73 was higher than that of patients with negative expressions $(\mathrm{P}<0.05)$. The expressions of VEGF and CD73 in RCC tissues were significantly positively correlated with MVD count $(\mathrm{P}<0.05)$. The five-year mortality rate of patients with positive expressions of VEGF and CD73 was higher than that of patients with negative expressions $(\mathrm{P}<0.05)$.

Conclusions: The expressions of VEGF and CD73 in RCC tissues can reflect the degree of tumor malignancy, invasion, and metastasis, and are closely related to the formation of microvessels in tumor tissues and the poor prognosis of patients.

Keywords: Renal cell carcinoma (RCC); vascular endothelial growth factor (VEGF); Ecto-5'-nucleotidase; microvessel density (MVD)

Submitted Apr 05, 2020. Accepted for publication May 29, 2020.

doi: $10.21037 /$ tau-20-904

View this article at: http://dx.doi.org/10.21037/tau-20-904 


\section{Introduction}

Renal cell carcinoma (RCC), a tumor originating from renal tubular epithelial cells, has the second highest incidence of any adult urogenital tumor. However, fewer than $15 \%$ of RCC patients have observable local symptoms, and, in most cases, patients exhibit no obvious symptoms in the early or even late stages of the disease, which seriously affects the prognosis (1). Tumor neovascularization is a key process in tumor proliferation, invasion, and metastasis. Microvessel density (MVD) can be indicative of the number of blood vessels in tumor tissues and has a close relationship with tumor prognosis (2). The diagnosis and prognosis of RCC are related to a variety of molecular markers, including molecular of tissue source, serum source and urine source, which have been extensively and deeply studied by scholars. Vascular endothelial growth factor (VEGF), as a pro-angiogenic factor, can promote angiogenesis by changing the extracellular matrix and increasing the permeability of blood vessels. And it may be related to MVD (3). Once the tumor occurs, expression of VEGF will be activated and secreted periodically, resulting in increased vascular permeability, plasma protein extravasation and fibrin aggregation of the tumor, further maintaining the infinite growth of the tumor and providing a basis for tumor invasion and metastasis (4). Multiple studies have shown that VEGF is an independent predictor of the prognosis of RCC patients (5). Extracellular 5'-nucleotidase (CD73) is a key enzyme in the production of extracellular adenosine. Highly expressed in a wide range of tumor tissues, CD73 participates in tumor immunosuppression and influences prognosis (6). CD73 has been shown in recent studies to be highly expressed in lymphatic lymphocytes and vascular cavity cells, which can enhance the vascular endothelial barrier function, induce angiogenesis. It is also closely related to tumor neovascularization (7). This study aimed to analyze the expression of vascular VEGF and CD73 and their relationship with clinical pathology, microvessel density (MVD), and prognosis in RCC, and to explore the mechanism of high VEGF and CD73 expression levels in cancer tissues, and their significance and value in treating RCC.

We present the following article in accordance with the STROBE reporting checklist (available at http://dx.doi. org/10.21037/tau-20-904).

\section{Methods}

\section{General information}

The clinical data of 76 patients with RCC who underwent radical nephrectomy between October, 2011 and October, 2013 in the Affiliated Hospital of Chengdu University of Traditional Chinese Medicine were retrospectively analyzed, and the postoperative paraffin specimens were collected. Patients were included if they: (I) had not received antitumor treatment before surgery; (II) had RCC pathologically confirmed after surgery; (III) were not lost to follow-up after surgery; and (IV) had well-preserved paraffin specimens. Patients were excluded if they: (I) had undergone radiotherapy or chemotherapy or taken other antitumor drugs before surgery; (II) had other serious diseases, such as dysfunction of important organs; or (III) had incomplete clinical data.

Of the 76 included patients, 46 were male and 30 were female. The patients were aged between 28 and 67 years old, with an average age of $56.24 \pm 11.36$ years. Left-sided lesions were found in 42 cases, while 34 cases had rightsided lesions. Based on WHO pathological grade, 30, 29, 13 , and 4 cases were G1, G2, G3, and G4, respectively, and 24, 26, 19 and 7 cases were Robson clinical stage I, II, III, and IV, respectively. There were 17 cases of lymph node metastasis.

\section{Detection of VEGF and CD73 by immunohistochemistry (IHC)}

Mouse anti-human VEGF monoclonal antibody, rabbit anti-human CD73 polyclonal antibody, rabbit anti-human CD34 polyclonal antibody, streptavidin staining kit, and diaminobiphenyl (DAB) color developing kit were all purchased from Beijing Boaosen Biotechnology Co. Ltd. (Beijing, China).

The specimens were fixed, dehydrated, and paraffinized. Followed by being sliced at $4 \mu \mathrm{m}$ using a microtome, dried in an incubator, conventionally dewaxed with water, antigen-repaired by EDTA. The specimens were treated with $3 \%$ hydrogen peroxide in order to remove endogenous peroxidase, and blocked in serum solution for $10 \mathrm{~min}$. Then, primary antibody was added and the specimens were incubated at $4{ }^{\circ} \mathrm{C}$ overnight. Biotin-labeled secondary antibody, horseradish-labeled streptavidin, and $\mathrm{DAB}$ coloration were added sequentially, before the specimens were counterstained with hematoxylin, dehydrated with gradient ethanol, rendered transparent with xylene, and mounted with neutral balsam. A breast cancer specimen was used as a positive control, and PBS was used in place of the primary antibody as a negative control.

The positive expression of VEGF and CD73 was 
Table 1 Expression of VEGF and CD73 in RCC

\begin{tabular}{lcc}
\hline Content & VEGF & CD73 \\
\hline Positive expression & 27 & 20 \\
- & 17 & 19 \\
+ & 21 & 24 \\
++ & 11 & 13 \\
+++ & $5.13 \pm 0.74$ & $7.48 \pm 1.16$ \\
mRNA expression & $0.96 \pm 0.18$ & $1.53 \pm 0.22$ \\
Protein expression & & \\
\hline
\end{tabular}

evaluated based on the standard of immune response score (IRS). Each sample was randomly observed in 5 magnification fields $(\times 400)$, with 100 cells counted in each field. First, the tumor cell staining intensity (SI) score was performed: 0 points for colorless, 1 point for light yellow, 2 points for brown-yellow, and 3 points for tan. The images were then scored according to the percentage of positive cells (PP): 0 points for negative, 1 point for $\leq 10 \%$ positive cells, 2 points for $11-50 \%$ positive cells, 3 points for $51-75 \%$ positive cells, and 4 points for $>75 \%$ positive cells. The final staining score was IRS $=\mathrm{SI} \times \mathrm{PP}$, recorded as negative: 0 points (-), weak positive: 1 to 4 points $(+)$, medium positive: 5 to 8 points $(++)$, and strong positive: 9 to 12 points $(+++)(8,9)$.

\section{MVD detection by IHC}

The tumor microvessels were stained with CD34. Brownyellow positively stained endothelial cells and cell clusters were recorded as one microvessel. First, five microvascular dense areas were selected under low magnification and the microvessels were counted under high magnification. MVD was calculated based on the average number of microvessels in these five areas (10).

\section{Real-time quantitative PCR (RT-qPCR)}

The total RNA in RCC tissues was extracted using the TRIzol kit, and the purity and concentration of it was determined by spectrophotometer. The cDNA synthesized using the reverse transcription kit, according to the instructions to reverse transcribe $1 \mu \mathrm{g}$ of RNA for each reaction. And then using RT-PCR instrument (Roche, Switzerland) for PCR amplification and followed by drawing dissolution curve after the amplification. The relative expression of VEGF and CD73 on the mRNA levels were calculated as $2^{-\Delta \Delta \mathrm{Ct}}$.

\section{Western blotting}

The protein expression of VEGF and CD73 was detected by Western blotting. First, $30 \mathrm{mg}$ tissue was taken, crushed, and lysed with RIPA lysis (supplemented with an appropriate amount of protease or phosphatase inhibitor), and ultrasound to obtain supernatant. After then quantified the concentration using BCA kit, the proteins of equal concentration were separated by the SDS-PAGE gel electrophoresis, transferred onto the membrane, incubated with primary and secondary antibodies, and finally detected using the ECL reagent. The relative protein expression levels of VEGF and CD73 were calculated by semiquantitative analysis with gel analyzer.

\section{Statistical methods}

All statistical analyses were performed using SPSS 20.0 software [IBM Corporation (SPSS Co.)]. Categorized data of VEGF and CD73 expression were expressed as n (\%). Nonranked data was analyzed by chi-square test, and rank data by rank-sum test. Continuous data such as MVD were expressed as mean $\pm \mathrm{SD}(\bar{x} \pm \mathrm{s})$ and analyzed using $t$-test. The correlation between the expression of VEGF and CD73 and MVD was analyzed by Spearman's correlation analysis. Results with $\mathrm{P}<0.05$ were considered to be statistically significant.

All procedures performed in this study were in accordance with the Declaration of Helsinki and the study was approved by Chengdu University of Traditional Chinese Medicine (No. SYXK-20200037). Because of the retrospective nature of the research, the requirement for informed consent was waived.

\section{Results}

\section{The expression of VEGF and CD73 in RCC tissues}

The positive expression of VEGF and CD73 in RCC by IHC and the relative expression on mRNA and protein levels were shown in Table 1.

\section{The relationship between the expression of VEGF and CD73 and the pathological grade of RCC}

There were significant differences in the expression 
Table 2 Relationship between the expression of VEGF and CD73 and RCC pathological features (n)

\begin{tabular}{|c|c|c|c|c|c|c|c|c|c|}
\hline Items & $\mathrm{n}$ & \multicolumn{4}{|c|}{ VEGF } & \multicolumn{4}{|c|}{ CD73 } \\
\hline \multicolumn{10}{|c|}{ Pathological grading } \\
\hline G1-G2 & 59 & 25 & 15 & 13 & 6 & 19 & 17 & 16 & 7 \\
\hline G3-G4 & 17 & 2 & 2 & 8 & 5 & 1 & 2 & 8 & 6 \\
\hline$P$ & - & \multicolumn{4}{|c|}{0.002} & \multicolumn{4}{|c|}{0.001} \\
\hline
\end{tabular}
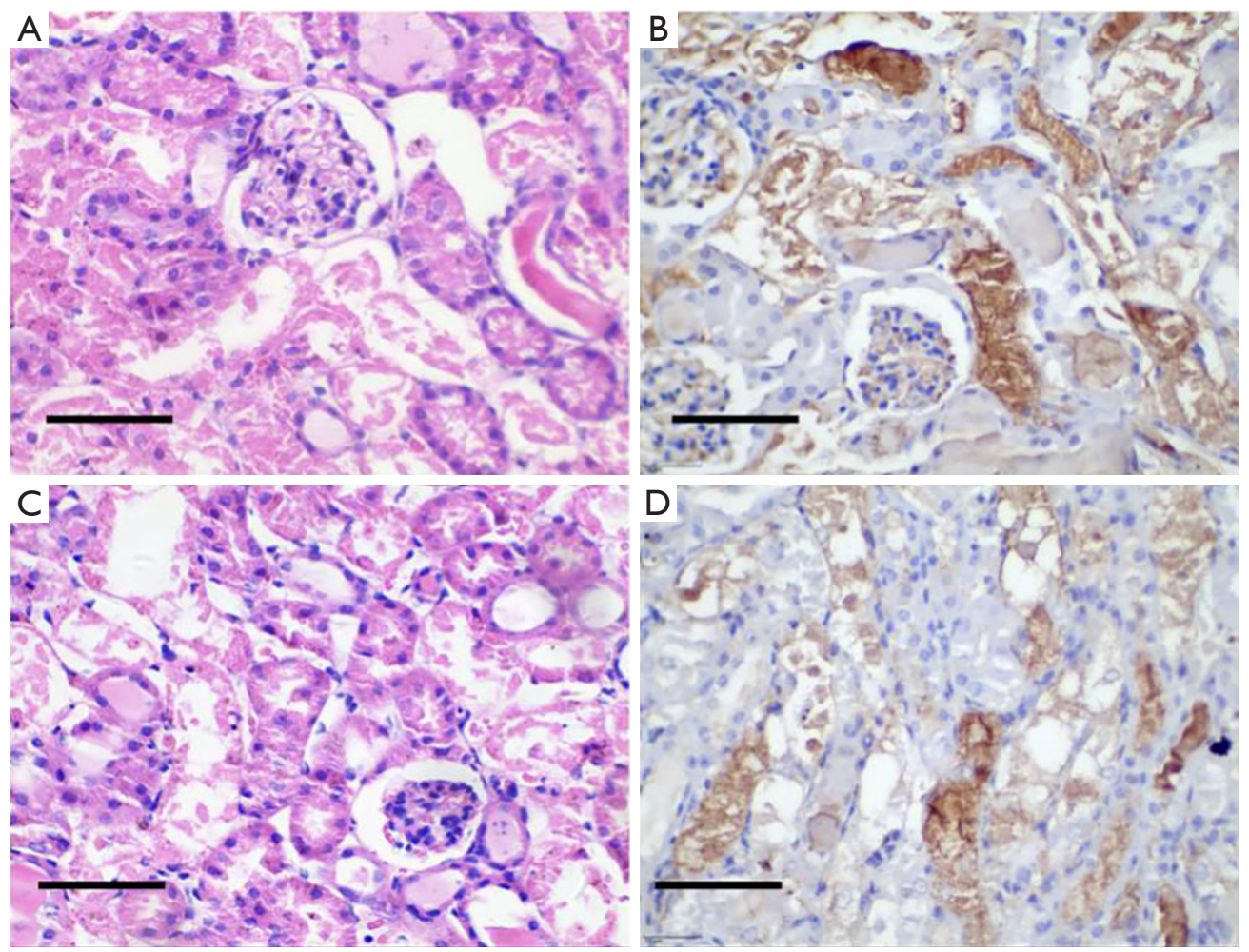

Figure 1 The expression of VEGF and CD73 in RCC, Hematoxylin-Eosin dyeing method. (A) VEGF negative expression in RCC (SP $\times 400)$; (B) VEGF positive expression in RCC (SP ×400); (C) CD73 negative expression in RCC $(\mathrm{SP} \times 400)$; (D) CD73 positive expression in RCC $(\mathrm{SP} \times 400)$. VEGF, vascular endothelial growth factor; RCC, renal cell carcinoma.

of VEGF and CD73 in tumor tissues of patients with different pathological grades of RCC $(\mathrm{P}<0.05$, Table 2$)$. The negative expression of VEGF in the tumor tissues and was almost colorless (Figure 1A). Positive VEGF expression was observed in the cell cytoplasm stained brown or light yellow and the cell membrane stained brown-yellow or tan (Figure $1 B$ ). The negative expression of CD73 in the tumor tissues was almost unstained (Figure 1C), and most of the positive expression staining was localized on the cell membrane, represented by brownish-yellow or yellow particles (Figure 1D).

\section{The relationship between the expression of VEGF and CD73 and RCC clinical stage}

There were significant differences in the expression of VEGF and CD73 in the tumor tissues of patients with different stages of RCC. The positive expression rates 
Table 3 Relationship between the expression of VEGF and CD73 and clinical stages of RCC (n)

\begin{tabular}{|c|c|c|c|c|c|c|c|c|c|}
\hline Items & $\mathrm{n}$ & \multicolumn{4}{|c|}{ VEGF } & \multicolumn{4}{|c|}{ CD73 } \\
\hline \multicolumn{10}{|c|}{ Clinical stages } \\
\hline$I-I I$ & 50 & 23 & 13 & 10 & 4 & 18 & 14 & 14 & 4 \\
\hline III-IV & 26 & 4 & 4 & 11 & 7 & 2 & 5 & 10 & 9 \\
\hline$P$ & - & \multicolumn{4}{|c|}{0.001} & \multicolumn{4}{|c|}{0.000} \\
\hline
\end{tabular}

VEGF, vascular endothelial growth factor; RCC, renal cell carcinoma.

Table 4 Relationship between the expression of VEGF and CD73 and RCC lymph node metastasis (n)

\begin{tabular}{|c|c|c|c|c|c|c|c|c|c|}
\hline Items & $\mathrm{n}$ & \multicolumn{4}{|c|}{ VEGF } & \multicolumn{4}{|c|}{ CD73 } \\
\hline \multicolumn{10}{|c|}{ Lymphatic metastasis } \\
\hline With & 17 & 2 & 1 & 7 & 7 & 1 & 1 & 8 & 7 \\
\hline Without & 59 & 25 & 16 & 14 & 4 & 19 & 18 & 16 & 6 \\
\hline$P$ & - & \multicolumn{4}{|c|}{0.001} & \multicolumn{4}{|c|}{0.000} \\
\hline
\end{tabular}

VEGF, vascular endothelial growth factor; RCC, renal cell carcinoma.

of VEGF and CD73 in RCC patients with stages III-IV were higher than those in patients with stages I-II $(\mathrm{P}<0.05$, Table 3).

\section{The relationship between the expression of VEGF and CD73 and RCC lymph node metastasis}

The expression of VEGF and CD73 in the tumor tissues of RCC patients with lymph node metastasis was significantly different from those without lymph node metastasis. The positive expression rates of VEGF and CD73 in patients with lymph node metastasis were significantly higher than in those without $(\mathrm{P}<0.05$, Table 4$)$.

\section{Correlation between VEGF and CD73 expression and MVD}

The MVD count of RCC patients with positive expressions of VEGF and CD73 was higher than that of patients with negative expressions $(\mathrm{P}<0.05$, Table 5). Moreover, the expression level of VEGF and CD73 in RCC tissues were significantly positively correlated with MVD count $(r=0.734$,
$0.561, \mathrm{P}<0.05)$

\section{Follow-up situation}

As of October 30, 2018, after 5 years of outpatient or telephone follow-up, 45 patients had survived and 31 patients had died. The causes of death were all related to the recurrence or metastasis of the primary tumor.

\section{Correlation between the expression of VEGF and CD73 and prognosis}

There was a statistically significant correlation between the expression of VEGF and CD73 and the prognosis of patients $\left(\chi^{2} \mathrm{VEGF}=7.165, \chi^{2} \mathrm{CD} 73=5.304 ; \mathrm{P}<0.05\right.$, Table 6$)$.

\section{Discussion}

As revealed by a study in 2018, the incidence of RCC in China in 2014 was approximately 4.99/100,000, and was accompanied by a mortality rate of $1.87 / 100,000$. Despite the rates of morbidity and mortality not being 
Table 5 Differences in MVD between patients with positive and negative expressions of VEGF and CD73 ( $\bar{x}_{ \pm}$s)

\begin{tabular}{|c|c|c|c|c|c|c|c|c|}
\hline Items & \multicolumn{4}{|c|}{ VEGF } & \multicolumn{4}{|c|}{ CD73 } \\
\hline MVD & $44.38 \pm 13.81$ & $73.45 \pm 10.37$ & 10.371 & 0.000 & $47.94 \pm 16.03$ & $73.78 \pm 12.07$ & 7.515 & 0.000 \\
\hline
\end{tabular}

VEGF, vascular endothelial growth factor; MVD, microvessel density.

Table 6 Correlation between the expression of VEGF and CD73 and prognosis

\begin{tabular}{|c|c|c|c|c|c|c|}
\hline 5-year survival & \multicolumn{3}{|c|}{ VEGF } & \multicolumn{3}{|c|}{ CD73 } \\
\hline Death & 17 & 14 & 31 & 13 & 18 & 31 \\
\hline Survival & 10 & 35 & 45 & 7 & 38 & 45 \\
\hline Total & 27 & 49 & 76 & 20 & 56 & 76 \\
\hline
\end{tabular}

VEGF, vascular endothelial growth factor.

high, but the rates have increased significantly in recent years (11). RCC can easily metastasize and mostly does so through the lymphatic and hematological systems. Tumor neoangiogenesis supplies nutrients to support the proliferation of cancer cells in RCC, but also directly facilitates its invasion further (12).

VEGF, an endothelial cell-specific mitogen, specifically targets vascular endothelial cells and directly stimulates endothelial cell proliferation. With the ability to produce collagenase and plasminogen activators, VEGF not only promotes tumor vascular network formation and provides sufficient nutrients for tumor proliferation, but also facilitates shedding of tumor cells into blood vessels or their infiltration into adjacent tissues (13). In this study, the positive rate of VEGF expression in RCC patients with grades G3-G4 was higher than that in patients with grade G1-G2, and the rates in RCC patients with stages III IV were higher than those in patients with stages I-II $(\mathrm{P}<0.05)$. Meanwhile, the rates of VEGF expression in RCC patients with lymph node metastasis were higher than those in patients without lymph node metastasis $(\mathrm{P}<0.05)$. These results are consistent with those of Sharma et al. (14). Moreover, the MVD count of RCC patients with positive VEGF expression was higher than that of patients with negative expression, and the expression level of VEGF was significantly positively correlated with the MVD count, which suggests the role high expression of VEGF plays in tumor angiogenesis.

Tumor angiogenesis is a key factor of supporting tumor growth. The vascular network can be developed and maintained by the continuous expression of VEGF, which can promote tumor growth and metastasis (15). Therefore, the results of this study indicated VEGF expression to be closely related to tumor malignancy, invasion ability, and metastasis in RCC. As a powerful vascular permeability factor, the ability of VEGF to enhance microvascular permeability is 50,000 times that of histamine (16). It can also increase the permeability of tumor blood vessels and cause extravasation of circulating macromolecules such as fibrin and plasma proteins, thus forming a fibrous extracellular matrix that can support fibroblasts and endothelial cells endogenously, and creating the basis substance of tumor cell growth and neovascularization (17). In the same study it was found that patients with high expression levels of VEGF accounted for a higher proportion of those with poor prognosis, and the 5-year mortality rate of VEGF-positive patients was significantly higher than that of VEGF-negative patients, which indicates that VEGF could serve as a predictor of poor prognosis in patients with RCC. VEGF overexpression reflects the aggressiveness and degree of active growth of the tumor, promotes neovascularization, and paves an easier path for the local recurrence and distant metastasis of tumors (18).

This study showed that the positive expression rate of CD73 was higher in RCC patients with grades G3-G4, stages III-IV, and lymph node metastasis, which suggests that CD73 expression is also closely related to tumor malignancy, invasion ability, and metastasis in RCC. Adenosine, which is found in abundance in the tumor microenvironment, is a key contributor to anti-tumor immunosuppression. 
CD73 is pivotal in the production of adenosine and shows significant immunosuppressive activity (19). As well as hypoxia and adenosine-rich conditions, other important factors in the upregulation of CD73 expression in tumor tissues include high levels of transforming growth factor $\beta$ (TGF $\beta$ ), interferon, and tumor necrosis factor (TNF $\alpha)$ in the tumor microenvironment (20). The results of a study by Ghalamfarsa $e t a l$. evidenced the close relationship between CD73 and tumor neovascularization, and showed that its high expression in lymphatic lymphocytes and vascular lumen cells could enhance vascular endothelial barrier function and induce angiogenesis, while less angiogenesis occurred in CD73-deficient mice (21). In this study, the MVD count of patients with positive CD73 expression was higher than that of patients with negative CD73 expression, and it was significantly positively correlated with the expression level of CD73. This indicates that the expression of CD73 in tumor tissue of patients with RCC could also reflect the microvascular formation status of a tumor. A similar phenomenon has also been discovered in non-small cell lung cancer tissues, which suggests that CD73 may be related to the neovascularization of various malignancies (22). It has been observed in previous literature that cells with CD73 high expression show more resistance to radiation and DNA-damaging agents (23). Therefore, the high expression of CD73 in RCC tumor tissues might not only influence tumor immunosuppression, development, metastasis, and tolerance to chemoradiotherapy, but might also promote tumor neovascularization, resulting in tumor proliferation, development, and deterioration, thus affecting patient prognosis. This study also showed that patients with positive CD73 expression had a higher five-year mortality rate and a poor prognosis. Therefore, CD73 could be used to evaluate the poor prognosis of patients with RCC. However, there are still limitations in this study. The number of cases included is small and it is a single-center study, so more cases need to be included in the follow-up study to further support the above conclusions.

In summary, the expression of VEGF and CD73 in RCC tumor tissues can reflect the degree of malignancy of tumors as well as their invasion and metastasis ability. VEGF and CD73 are closely related to microvessel formation in cancer tissues and the poor prognosis of patients, and could provide great value in the evaluation of RCC prognosis.

\section{Acknowledgments}

Funding: None.

\section{Footnote}

Reporting Checklist: The authors have completed the STROBE reporting checklist. Available at http://dx.doi. org/10.21037/tau-20-904

Data Sharing Statement: Available at http://dx.doi. org/10.21037/tau-20-904

Conflicts of Interest: All authors have completed the ICMJE uniform disclosure form (available at http://dx.doi. org/10.21037/tau-20-904). The authors have no conflicts of interest to declare.

Ethical Statement: The authors are accountable for all aspects of the work in ensuring that questions related to the accuracy or integrity of any part of the work are appropriately investigated and resolved. All procedures performed in this study were in accordance with the Declaration of Helsinki and the study was approved by Chengdu University of Traditional Chinese Medicine (No. SYXK-20200037). Because of the retrospective nature of the research, the requirement for informed consent was waived.

Open Access Statement: This is an Open Access article distributed in accordance with the Creative Commons Attribution-NonCommercial-NoDerivs 4.0 International License (CC BY-NC-ND 4.0), which permits the noncommercial replication and distribution of the article with the strict proviso that no changes or edits are made and the original work is properly cited (including links to both the formal publication through the relevant DOI and the license). See: https://creativecommons.org/licenses/by-nc-nd/4.0/.

\section{References}

1. Trpkov K, Siadat F. Immunohistochemical screening for the diagnosis of succinate dehydrogenase-deficient renal cell carcinoma and fumarate hydratase-deficient renal cell carcinoma. Ann Transl Med 2019;7:S324.

2. Jilaveanu LB, Puligandla M, Weiss SA, et al. Tumor Microvessel Density as a Prognostic Marker in HighRisk Renal Cell Carcinoma Patients Treated on ECOG- 
ACRIN E2805. Clin Cancer Res 2018;24:217-23.

3. Rathi N, Maughan BL, Agarwal N, et al. The tango of immunotherapy and targeted therapy in metastatic renal cell carcinoma. Transl Cancer Res 2019;8:E1-6.

4. Morgantetti G, Ng KL, Samaratunga H, et al. Prostate specific membrane antigen (PSMA) expression in vena cava tumour thrombi of clear cell renal cell carcinoma suggests a role for PSMA-driven tumour neoangiogenesis. Transl Androl Urol 2019;8:S147-55.

5. Mikami S, Mizuno R, Kondo T, et al. Clinical significance of programmed death-1 and programmed death-ligand 1 expression in the tumor microenvironment of clear cell renal cell carcinoma. Cancer Sci 2019;110:1820-8.

6. Stitzlein L, Rao P, Dudley R. Emerging oral VEGF inhibitors for the treatment of renal cell carcinoma. Expert Opin Investig Drugs 2019;28:121-30.

7. Ren ZH, Yuan YX, Ji T, et al. Research progress on the mechanism of CD73 in malignant tumors. Journal of Practical Stomatology 2017;33:404-8.

8. Kong LS, Chai KX, Chen YQ. Determination and significance of monocyte chemotactic protein- 3 and microvessel density in muscular tissue of patients with dermatomyositis and polymyositis. The Journal of Practical Medicine 2017;33:1113-6.

9. Yu MS, Yan XL, Zhang XB, et al. Expressions of endocan, vascular endothelial growth factor and matrix metalloproteinase- 9 and their significances in pituitary adenomas apoplexy. Chinese Journal of Neuromedicine 2018;17:222-6.

10. Sierko E, Wojtukiewicz M, Zimnoch L, et al. Expression of tissue factor pathway inhibitor (TFPI) in human breast and colon cancer tissue. Thromb Haemost 2010;103:198-204.

11. Liu SZ, Guo LW, Cao XQ, et al. Estimation on the incidence and mortality of kidney cancer in China, in 2014. Zhonghua Liu Xing Bing Xue Za Zhi 2018;39:1346-50.

12. Mollica V, Di Nunno V, Massari F. Pembrolizumab plus axitinib: a new treatment option for patients with metastatic renal cell carcinoma. Chin Clin Oncol 2019;8:S21.

13. Liu R, Pei Q, Li W, et al. Renal Cell Carcinoma: Relationship between Level of Vascular Endothelial Growth Factor in Serum of Patients and Clinical Pathological Stage. Progress in Modern Biomedicine 2016;16:4065-7.

14. Sharma K, Patidar K, Ali MA, et al. Structure-Based Virtual Screening for the Identification of High Affinity
Compounds as Potent VEGFR2 Inhibitors for the Treatment of Renal Cell Carcinoma. Curr Top Med Chem 2018;18:2174-85.

15. Wang J, Li CT, Cao WY. Expression of vascular endothelial growth factor and its vital catalytic role in promotion of invasion and metastasis of hepatocellular carcinoma cells. Chinese Journal of Microbiology and Immunology 2019;39:73-8.

16. Jiang YX, Zhou X, Yu WJ, et al. Expression and their relationship with clinicopathological characteristics of CD147 and VEGF in renal cell carcinoma. Chinese Journal of Clinical and Experimental Pathology 2016;32:757-60.

17. Li J, Liu DH, Zou N,et al. Expression of HIF-2 $\alpha$, VEGF, COX-2, MMP-9 and Their Relationship with Renal Cell Carcinoma Angiogenesis. Labeled Immunoassays and Clinical Medicine 2017;24:1243-6.

18. Li XP, Ren KM, Zhang L, et al. Expression of tumor necrosis factor wuper family 15 , vascular endothelial growth factor and Survivin in non- small cell lung cancer and correlation with long term survival time. Chinese Journal of Experimental Surgery 2018;35:1793-6.

19. Ma SY, Wang YT, Di N, et al. The expression of CD73 in epithelial ovarian tumor and relevant research of tumor growth and transfer. Chinese Journal of Laboratory Diagnosis 2016;20:2037-40.

20. Chang JJ, Ji ZY, Yan CH, et al. Biological characteristics of lung cancer-derived mesenchymal stem cells. Chinese Journal of Cancer Prevention and Treatment 2018;25:91-8.

21. Ghalamfarsa G, Kazemi MH, Raoofi Mohseni S, et al. CD73 as a potential opportunity for cancer immunotherapy. Expert Opin Ther Targets.2019;23:127-42.

22. Yang XC, Li DT, Gao YJ, et al. Relationship between CD73 expression and microvessel density in non-small cell lung cancer and its effect on clinical prognosis. Chinese Journal of Cancer Prevention and Treatment 2018;25:99-105.

23. Song L, Ye W, Cui Y, et al. Ecto-5'-nucleotidase (CD73) is a biomarker for clear cell renal carcinoma stem-like cells. Oncotarget 2017;8:31977-92.

Cite this article as: Mei $\mathrm{X}$, Shu J, Huang R, Chu X, Tian Y. Expression of VEGF, CD73 and their relationship with clinical pathology, microvessel density, and prognosis in renal cell carcinoma. Transl Androl Urol 2020;9(3):1366-1373. doi:10.21037/tau-20-904 